\section{Dificuldade auditiva autorreferida e exposição ocupacional a agentes otoagressores: um estudo de base populacional}

\author{
Self-reported hearing impairment and \\ occupational exposure to hazardous noise and \\ ototoxic agents: a population-based study
}

\section{Dificultad auditiva autoinformada y exposición ocupacional a agentes otoagresores: un estudio de base poblacional}

\section{Resumo}

O objetivo deste estudo foi estimar a associação entre dificuldade auditiva autorreferida e exposição ocupacional a agentes otoagressores em trabalhadores brasileiros. Trata-se de um estudo transversal realizado com dados da Pesquisa Nacional de Saúde (PNS-2013). A variável dependente foi a dificuldade auditiva autorreferida, e foram analisadas três exposições principais: ruído, poeira industrial e substâncias químicas. Realizou-se análise de regressão logística, estimando-se as odds ratio (OR) brutas e ajustadas, com intervalos de 95\% de confiança (IC95\%). As variáveis principais de exposição foram ajustadas entre si e pelas covariáveis sexo, idade, ambiente de trabalho, tempo de trabalho e hipertensão arterial. Participaram deste estudo 36.442 trabalhadores. Maior prevalência de dificuldade auditiva foi observada em indivíduos expostos à poeira industrial $(9,9 \%)(p<0,001)$. Além disso, quanto maior a idade do trabalhador e tempo de trabalho, maior foi a prevalência do desfecho $(p<0,001)$. Na análise ajustada, trabalhadores expostos a ruido apresentaram 1,65 vez mais chance de referir dificuldade auditiva, quando comparados aos indivíduos não expostos ( $p<0,001)$. O mesmo ocorreu com trabalhadores expostos à poeira industrial $(O R=1,36)(p=0,012)$. Não foi observada associação entre o desfecho e a variável exposição a substâncias químicas ( $p=$ 0,120). Observou-se associação entre dificuldade auditiva e exposição ocupacional a ruído e à poeira industrial em trabalhadores brasileiros. Reforçam-se a importância do aprimoramento de politicas públicas em saúde auditiva e o desenvolvimento de ações voltadas à prevenção e ao monitoramento auditivo em ambiente ocupacional.

Perda Auditiva; Saúde do Trabalhador; Inquéritos Epidemiológicos; Ruído; Poeira
Danúbia Hillesheim 1

Fernanda Zucki 2

Simone Mariotti Roggia 2

Karina Mary de Paiva 2

doi: 10.1590/0102-311X00202220

\author{
Correspondência \\ D. Hillesheim \\ Programa de Pós-graduação em Saúde Coletiva, Universidade \\ Federal de Santa Catarina. \\ Rua Delfino Conti s/ $n$, Campus Reitor João David Ferreira \\ Lima, Florianópolis, SC 88040-900, Brasil. \\ nubiah12@yahoo.com.br \\ 1 Programa de Pós-graduação em Saúde Coletiva, Universidade \\ Federal de Santa Catarina, Florianópolis, Brasil. \\ 2 Departamento de Fonoaudiologia, Universidade Federal de \\ Santa Catarina, Florianópolis, Brasil.
}




\section{Introdução}

De acordo com o estudo Global Burden of Disease, a perda auditiva é a quarta principal causa de incapacidade em todo o mundo 1. Estima-se que, em 2050, mais de 900 milhões de pessoas terão algum tipo de perda auditiva 2. Esse déficit pode ser ocasionado por problemas genéticos, patologias infecciosas, doenças crônicas do ouvido, uso de medicamentos ototóxicos e, principalmente, exposição a riscos ocupacionais 3 . Estudos apontaram que a prevalência de perda auditiva autorreferida em adultos $(\geq$ 18 anos) pode variar de 17 a 19\%, com gradativo aumento percentual em adultos mais velhos 4,5. Além disso, observando-se a estratificação etária, há baixo percentual de diagnóstico de deficiência auditiva em adultos jovens 6 .

A exposição a ruído é estabelecida mundialmente como um problema de saúde pública, podendo ocasionar a perda auditiva induzida por ruído (PAIR). Embora seja o mais prevalente, o ruído não é o único agente otoagressor, pois vibração 7,8 , substâncias químicas 7,8 e alguns tipos de poeira 7,9 são apresentados pela literatura como passíveis de ocasionar alterações no sistema auditivo. A PAIR, nessa condição, é reconhecida como um agravo à saúde relacionada ao trabalho, de notificação compulsória 10. Apesar de a PAIR ser considerada totalmente passível de prevenção, ainda acomete entre 7 e $21 \%$ dos trabalhadores, tendo os países em desenvolvimento os riscos mais elevados 11,12. A exposição a produtos químicos potencialmente tóxicos ao sistema auditivo tem como vias de introdução a via digestiva, cutânea e a respiratória, sendo essa última a via de introdução da poeira 7 .

A pluralidade das vias e formas de exposição no ambiente de trabalho, somada às questões inerentes ao próprio indivíduo exposto, como fatores genéticos e estilo de vida, são responsáveis pelo processo de adoecimento 13 . O conhecimento dos fatores de risco nos ambientes ocupacionais tem contribuído para o enfrentamento desse problema, por meio de mudanças no processo de trabalho e da adoção de medidas de controle ambiental, minimizando a exposição e conscientizando empregadores e trabalhadores, em busca da atenção integral à saúde do trabalhador 14,15,16,17,18. Pesquisas nacionais acerca da exposição ocupacional a agentes otoagressores são escassas 19, especialmente com dados de inquéritos populacionais de trabalhadores informais, desassistidos por esferas sindicais, governamentais e acadêmicas 20 .

Pesquisas recentes utilizaram dados de base populacional autorreferidos sobre percepção de riscos ocupacionais 20,21,22. Oenning et al. 21 observaram que os ambientes físico e psicossocial do trabalho podem estar associados à autoavaliação ruim de saúde. Soares et al. 22 consideraram os riscos ocupacionais como fatores de ajuste na associação encontrada entre deficiência auditiva e diabetes entre adultos no Brasil. Assunção et al. 20 encontraram uma prevalência de exposição a ruído em ambiente ocupacional variando de $21,9 \%$ a $40,9 \%$ nos estados brasileiros, com relatos de sintomas auditivos e extra-auditivos entre a população alvo.

Assim, entende-se que a vigilância das exposições ocupacionais é essencial na determinação do risco e da carga das doenças, porém, os métodos atuais de vigilância, muitas vezes, subestimam dados de morbimortalidade, o que tem estimulado o desenvolvimento de estudos de autopercepção dos trabalhadores em relação a problemas de saúde em larga escala 23,24,25. Destaca-se a importância do uso de dados de base populacional para o sistema nacional de saúde, tendo em vista que essas informações podem auxiliar no planejamento e monitoramento de ações voltadas à promoção, à prevenção e à proteção de agravos, além de contribuir para a análise de políticas públicas 26 , ressaltando-se os dados da presente pesquisa que podem subsidiar as ações voltadas à saúde auditiva.

Diante desse contexto, o objetivo deste estudo foi estimar a associação entre dificuldade auditiva autorreferida e exposição ocupacional a agentes otoagressores em trabalhadores brasileiros.

\section{Métodos}

\section{Delineamento do estudo e fonte de dados}

Trata-se de um estudo transversal analítico, realizado com dados do inquérito da Pesquisa Nacional de Saúde (PNS-2013). A PNS é uma pesquisa de abrangência nacional, desenvolvida pelo Ministério 
da Saúde e Instituto Brasileiro de Geografia e Estatística (IBGE). O objetivo geral da PNS é produzir dados em âmbito nacional sobre a situação de saúde e estilo de vida da população brasileira 27.

A PNS contou com a participação total de 205.546 adultos entrevistados em 60.202 domicílios brasileiros. O processo de amostragem foi feito por conglomerados e dividido em três estágios. Informações minuciosas sobre o processo de amostragem, bem como sobre o método para coleta de dados foram descritas anteriormente 27,28 .

O questionário aplicado por técnicos do IBGE foi composto por três partes: informações do domicílio, características gerais de todos os moradores do domicílio e informações de um morador adulto, com 18 anos ou mais de idade, selecionado com equiprobabilidade entre todos os residentes elegíveis. O questionário referente ao adulto forneceu a maioria das variáveis investigadas neste estudo. A amostra foi composta por trabalhadores adultos ( $\geq 18$ anos) que estavam trabalhando dentro da semana de referência, que responderam à questão E1 do Módulo E do questionário: "Na semana de 21 a 27 de julho de 2013, você trabalhou ou estagiou, durante pelo menos uma hora, em alguma atividade remunerada em dinheiro?", resultando em 36.442 trabalhadores brasileiros que poderiam estar formalmente ou informalmente empregados.

\section{Variável dependente}

A variável dependente foi a dificuldade auditiva autorreferida, obtida por meio da pergunta: "Em geral, que grau de dificuldade o(a) Sr(a) tem para ouvir?". Os indivíduos eram orientados: "Ao responder à próxima pergunta leve em conta o aparelho auditivo, se o(a) sr(a) utilizar". A pergunta apresentava as seguintes categorias de resposta: nenhum, leve, médio, intenso e não consegue. Para este estudo, as categorias leve, médio, intenso e não consegue foram categorizadas como dificuldade positiva para ouvir (sim), e os indivíduos que relataram "nenhum" foram considerados sem dificuldade auditiva (não). Essa pergunta consta no Módulo $\mathrm{N}$ do questionário (percepção do estado de saúde).

\section{Variáveis independentes}

As variáveis independentes são oriundas do Módulo $\mathrm{M}$ (outras características do trabalho e apoio social), sendo obtidas por meio das perguntas: "Pensando em todos os seus trabalhos, o(a) Sr(a) está em exposição a ruído (barulho intenso) que pode afetar a sua saúde?”; "Pensando em todos os seus trabalhos, o(a) Sr(a) está exposto(a) a manuseio de substâncias químicas que podem afetar a sua saúde?” e "Pensando em todos os seus trabalhos, o(a) Sr(a) está em exposição à poeira industrial (pó de mármore) que pode afetar a sua saúde?”. Diante dessas questões, construíram-se as variáveis de exposição: ruído (não; sim), poeira industrial (não; sim) e substâncias químicas (não; sim).

\section{Covariáveis}

As covariáveis foram: sexo (masculino; feminino), idade (18-39; 40-59; 60 anos ou mais), raça (branca; preta; amarela; parda; indígena), ambiente de trabalho (aberto; fechado; ambos), tempo total de trabalho em anos completos (0-4; 5-9; 10 ou mais) e hipertensão arterial autorreferida (não; sim), coletada por meio da pergunta: "algum médico já lhe deu o diagnóstico de hipertensão arterial (pressão alta)?".

\section{Análise de dados}

Inicialmente, foi realizada a análise descritiva de todas as variáveis, por meio do cálculo de frequências absolutas e relativas da amostra. Foram estimadas as prevalências de dificuldade auditiva segundo as variáveis de exposição e covariáveis da pesquisa, usando-se, para comparar as proporções, o teste de qui-quadrado de Pearson.

Tanto para a análise bruta (bivariada) quanto para a ajustada, a odds ratio (OR) foi utilizada como medida de associação, considerando-se, nas análises, os parâmetros amostrais e o delineamento do estudo (comando svy). Foi escolhida a técnica de regressão logística por se mostrar mais apropriada para eventos de baixa magnitude 29 , a partir da prevalência de dificuldade auditiva encontrada no presente estudo. 
As variáveis principais de exposição foram ajustadas entre si e pelas covariáveis que apresentaram valor de $\mathrm{p}<0,20$ na análise bruta (bivariada). As variáveis foram incluídas de forma simultânea na análise ajustada (método direto), com o intuito de avaliar o efeito dessas sobre o desfecho 30 . Consideraram-se significativas aquelas que apresentaram um valor de $\mathrm{p}<0,05$ quando ajustadas pelas demais. Optou-se por apresentar, na tabela da análise ajustada, apenas as variáveis principais de exposição do estudo. As análises foram conduzidas no software Stata, versão 14.0 (https://www.stata.com).

\section{Aspectos éticos}

A PNS foi aprovada pela Comissão Nacional de Ética em Pesquisa (CONEP), em 8 de julho de 2013, sob o número 10853812.7.0000.0008, do Conselho Nacional de Saúde (CNS). Todos os indivíduos que aceitaram participar da pesquisa assinaram um Termo de Consentimento Livre e Esclarecido (TCLE). Além disso, destaca-se que são dados de domínio público e sem a identificação dos participantes.

\section{Resultados}

Participaram deste estudo 36.442 trabalhadores. A maior proporção da amostra foi composta por homens $(53,4 \%)$, adultos mais jovens $(50,4 \%)$ e indivíduos autodeclarados pardos $(48,4 \%)$. Com relação ao ambiente ocupacional, 50,5\% dos trabalhadores possuíam entre 0 e 4 anos de trabalho, seguidos pelos indivíduos com 10 anos ou mais $(33,4 \%)$. Além disso, constatou-se maior prevalência de ambientes fechados de trabalho (50,5\%). Dentre as exposições ocupacionais mais prevalentes, 30,5\% estavam expostos a ruído, seguido pelas substâncias químicas $(17,3 \%)$ e poeira industrial (8,2\%). A dificuldade para ouvir foi referida por 6,8\% dos participantes (Tabela 1).

Houve maior prevalência de dificuldade para ouvir em homens (7,6\%) e idosos ( $\geq 60$ anos) (17\%). Além disso, quanto maior o tempo de trabalho (em anos), maior foi a prevalência de dificuldade auditiva $(8,9 \%)(\mathrm{p}<0,001)$. Também houve maior proporção do desfecho em trabalhadores expostos a ruído (8,9\%), quando comparados aos indivíduos que referiram não estar ( $\mathrm{p}<0,001)$. O mesmo ocorreu com indivíduos expostos à poeira industrial $(9,9 \%)$ e a substâncias químicas $(8,6 \%)(\mathrm{p}<0,001)$ (Tabela 1).

$\mathrm{Na}$ análise bruta, as três exposições principais estavam associadas ao desfecho ( $<0,001)$. Observou-se razão de chances de 1,64 (IC95\%: 1,41-1,90) para o ruído, 1,37 (IC95\%: 1,16-1,63) para as substâncias químicas e 1,66 (IC95\%: 1,35-2,05) para a poeira industrial (Tabela 2).

$\mathrm{Na}$ análise ajustada, trabalhadores expostos a ruído apresentaram 1,65 (IC95\%: 1,40-1,94) vez mais chance de referir dificuldade auditiva, quando comparados aos indivíduos não expostos a ruído $(\mathrm{p}<0,001)$. O mesmo ocorreu com trabalhadores expostos à poeira industrial (OR = 1,36; IC95\%: 1,06-1,74). Não foi observada associação estatisticamente significante entre dificuldade auditiva e exposição a substâncias químicas (IC95\%: 0,96-1,37) (Tabela 3).

\section{Discussão}

A prevalência de dificuldade auditiva autorreferida encontrada neste estudo foi de 6,8\%, com maior prevalência em homens, idosos, expostos à poeira industrial, a ruído e a substâncias químicas. Quanto maior a idade do trabalhador e o tempo de trabalho, maior foi a prevalência do desfecho. Observou-se associação estatisticamente significante entre dificuldade auditiva autorreferida e exposição ocupacional a ruído e à poeira industrial em trabalhadores brasileiros. Não foi observada associação com a variável substâncias químicas.

Poucas pesquisas investigaram a prevalência de dificuldade auditiva autorreferida entre trabalhadores 31,32,33,34 e, quando realizadas, apresentavam métodos distintos, dificultando a comparação entre os achados deste estudo. Contudo, a prevalência de 6,8\% de dificuldade auditiva encontrada é inferior aos resultados do inquérito National Health Interview Survey (NHIS) dos Estados Unidos, com prevalência de 10,5\% de dificuldade auditiva autorreferida dentre 14.453 trabalhadores de diversas áreas 32 . 
Tabela 1

Caracterização e prevalência de dificuldade auditiva segundo características da amostra. Pesquisa Nacional de Saúde, 2013.

\begin{tabular}{|c|c|c|c|c|}
\hline Variável & $\mathbf{n}$ & $\%$ & $\begin{array}{l}\text { Dificuldade } \\
\text { auditiva (\%) }\end{array}$ & Valor de $p$ * \\
\hline Sexo & & & & $<0,001$ \\
\hline Masculino & 19.450 & 53,4 & 7,6 & \\
\hline Feminino & 16.992 & 46,6 & 6,0 & \\
\hline Idade (anos) & & & & $<0,001$ \\
\hline $18-39$ & 19.841 & 50,4 & 4,3 & \\
\hline $40-59$ & 14.078 & 38,6 & 8,6 & \\
\hline 60 ou mais & 2.523 & 7,0 & 17,0 & \\
\hline Raça & & & & 0,003 \\
\hline Branca & 14.744 & 40,5 & 6,6 & \\
\hline Preta & 3.462 & 9,5 & 7,9 & \\
\hline Amarela & 308 & 0,9 & 6,1 & \\
\hline Parda & 17.681 & 48,4 & 6,8 & \\
\hline Indígena & 247 & 0,7 & 11,7 & \\
\hline Tempo de trabalho (anos) & & & & $<0,001$ \\
\hline $0-4$ & 18.413 & 50,5 & 5,8 & \\
\hline $5-9$ & 5.845 & 16,1 & 5,9 & \\
\hline 10 ou mais & 12.184 & 33,4 & 8,9 & \\
\hline Ambiente de trabalho & & & & $<0,001$ \\
\hline Fechado & 18.413 & 50,5 & 6,0 & \\
\hline Aberto & 10.673 & 29,3 & 7,6 & \\
\hline Ambos & 7.356 & 20,2 & 7,9 & \\
\hline Hipertensão arterial & & & & $<0,001$ \\
\hline Não & 29.437 & 83,4 & 6,0 & \\
\hline Sim & 5.853 & 16,6 & 11,3 & \\
\hline Ruído & & & & $<0,001$ \\
\hline Não & 25.322 & 69,5 & 6,0 & \\
\hline $\operatorname{Sim}$ & 11.120 & 30,5 & 8,9 & \\
\hline Substâncias químicas & & & & $<0,001$ \\
\hline Não & 30.129 & 82,7 & 6,5 & \\
\hline $\operatorname{Sim}$ & 6.313 & 17,3 & 8,6 & \\
\hline Poeira industrial & & & & $<0,001$ \\
\hline Não & 33.462 & 91,8 & 6,6 & \\
\hline Sim & 2.980 & 8,2 & 9,9 & - \\
\hline \multicolumn{5}{|l|}{ Dificuldade auditiva } \\
\hline Não & 33.944 & 93,2 & - & \\
\hline Sim & 2.498 & 6,8 & - & \\
\hline
\end{tabular}

* Valor de p obtido por meio do teste qui-quadrado de Pearson. 
Tabela 2

Análise de regressão logística bruta entre agentes otoagressores, demais características da amostra e dificuldade auditiva. Pesquisa Nacional de Saúde, 2013.

\begin{tabular}{|c|c|c|}
\hline \multirow[t]{2}{*}{ Variáveis } & \multicolumn{2}{|c|}{ Dificuldade auditiva } \\
\hline & OR bruta (IC95\%) & Valor de $p$ * \\
\hline Sexo & & 0,002 \\
\hline Masculino & 1,00 & \\
\hline Feminino & $0,78(0,67-0,91)$ & \\
\hline Idade (anos) & & $<0,001$ \\
\hline $18-39$ & 1,00 & \\
\hline $40-59$ & $2,27(1,92-2,68)$ & \\
\hline 60 ou mais & $4,99(3,98-6,27)$ & \\
\hline Raça & & 0,971 \\
\hline Branca & 1,00 & \\
\hline Preta & $1,10(0,83-1,45)$ & \\
\hline Amarela & $0,76(0,39-1,50)$ & \\
\hline Parda & $0,99(0,85-1,15)$ & \\
\hline Indígena & $1,94(1,00-3,76)$ & \\
\hline Tempo de trabalho (anos) & & $<0,001$ \\
\hline $0-4$ & 1,00 & \\
\hline $5-9$ & $1,22(0,99-1,53)$ & \\
\hline 10 ou mais & $1,67(1,43-1,96)$ & \\
\hline Ambiente de trabalho & & $<0,001$ \\
\hline Fechado & 1,00 & \\
\hline Aberto & $1,27(1,07-1,52)$ & \\
\hline Ambos & $1,41(1,17-1,69)$ & \\
\hline Hipertensão arterial & & $<0,001$ \\
\hline Não & 1,00 & \\
\hline Sim & $1,95(1,63-2,32)$ & \\
\hline Ruído & & $<0,001$ \\
\hline Não & 1,00 & \\
\hline Sim & $1,64(1,41-1,90)$ & \\
\hline Substâncias químicas & & $<0,001$ \\
\hline Não & 1,00 & \\
\hline Sim & $1,37(1,16-1,63)$ & \\
\hline Poeira industrial & & $<0,001$ \\
\hline Não & 1,00 & \\
\hline Sim & $1,66(1,35-2,05)$ & \\
\hline
\end{tabular}

IC95\%: intervalo de 95\% de confiança; OR: odds ratio.

* Valor de p obtido por meio da análise de regressão logística bruta.

Maior prevalência do desfecho também foi observada em indivíduos com idade maior ou igual a 60 anos e em indivíduos do sexo masculino. O fator idade é reconhecido como uma das principais causas de perda de audição, denominada age-related hearing loss (perda auditiva relacionada à idade) 35,36. A maior prevalência de perda auditiva em homens foi observada em todas as exposições ocupacionais avaliadas no estudo de carga global 37, assim como em estudos brasileiros 38,39. Tal fato pode ser explicado pelos homens ingressarem na força de trabalho mais cedo e estarem expostos a mais riscos ocupacionais, com possíveis implicações auditivas 40 .

A associação entre dificuldade auditiva e ruído era esperada. Os resultados encontrados estão em convergência com diversas pesquisas 11,41,42. Dentre os fatores associados à perda auditiva em um 
Tabela 3

Análise de regressão logística ajustada entre agentes otoagressores e dificuldade auditiva. Pesquisa Nacional de Saúde, 2013.

\begin{tabular}{|c|c|c|}
\hline \multirow[t]{2}{*}{ Variáveis } & \multicolumn{2}{|c|}{ Dificuldade auditiva } \\
\hline & OR ajustada * (IC95\%) & Valor de $p$ \\
\hline Ruído & & $<0,001$ \\
\hline Não & 1,00 & \\
\hline $\operatorname{Sim}$ & $1,65(1,40-1,94)$ & \\
\hline Substâncias químicas & & 0,120 \\
\hline Não & 1,00 & \\
\hline Sim & $1,15(0,96-1,37)$ & \\
\hline Poeira industrial & & 0,012 \\
\hline Não & 1,00 & \\
\hline Sim & $1,36(1,06-1,74)$ & \\
\hline
\end{tabular}

IC95\%: intervalo de 95\% de confiança; OR: odds ratio.

* Variáveis ajustadas entre si e por sexo, idade, ambiente, tempo de trabalho e hipertensão arterial.

estudo realizado na Coreia do Sul, destacou-se o ruído ocupacional com uma OR = 1,28, uma OR menor que a encontrada em nosso estudo 41.

A clássica definição da PAIR contempla a diminuição gradual da acuidade auditiva, de caráter irreversível 7. A sintomatologia abrange intercorrências auditivas (perda auditiva sensorioneural, zumbido, otalgia, intolerância a sons intensos e diminuição da inteligibilidade de fala) e não auditivas (estresse, irritação, distúrbios do sono, dificuldade de concentração e problemas cardiovasculares) 11,43. Sua fisiopatologia aponta para danos às células ciliadas da cóclea e a sinaptopatia 44. A destruição do órgão de Corti pode ser o resultado de dois mecanismos: destruição mecânica, por exposição curta a intensidades extremas de ruído ou descompensação metabólica, após exposição prolongada a ruído 45 .

Mesmo diante de seu caráter irreversível, a PAIR é considerada como o agravo auditivo mais prevenível 46. Os números, contudo, não são favoráveis, à medida que se estima que, das 466 milhões de pessoas no mundo que apresentam perda auditiva incapacitante, $16 \%$ possam ser atribuídas exclusivamente à exposição ocupacional a ruído 3,47 . Observa-se queda da incidência da PAIR em países industrializados, provavelmente devido ao estabelecimento de medidas preventivas 11 , aspecto não verificado em países em desenvolvimento, o que acarreta um impacto direto na qualidade da vida do trabalhador 48 . O estudo de Masterson et al. 32 apontou a prevalência de 23\% de dificuldade auditiva autorreferida, $15 \%$ de zumbido e $9 \%$ para ambas as variáveis entre os trabalhadores expostos a ruído ocupacional. Já os resultados de Kerns et al. 33 demonstraram que, dos 25\% dos trabalhadores que tinham histórico de exposição a ruído ocupacional, 12\% apresentavam dificuldade auditiva autorreferida, podendo ser atribuída à exposição a esse agente.

Com relação à poeira industrial, indivíduos expostos a ela apresentaram maiores chances de relatar dificuldade auditiva. O mecanismo responsável por essa associação pode estar relacionado ao fato de substâncias químicas, potencialmente ototóxicas, estarem presentes nas partículas de poeira. A poeira tem sido citada como meio de contaminação com outras substâncias químicas, tais como os pesticidas e o pó de chumbo 7; bem como causadora de problemas respiratórios 49,50,51,52,53,54, os quais podem ter influência no sistema auditivo 55,56. Além disso, em muitos ambientes de trabalho nos quais a poeira está presente, como construção civil 9 e marmorarias 57 , em geral, os níveis de ruído também são elevados.

Não foi encontrado nenhum estudo que tenha utilizado especificamente o termo "poeira industrial" nem "pó de mármore" relacionado a dificuldades auditivas. Foram constatadas alterações auditivas autorreferidas em trabalhadores expostos à poeira existente na construção civil 9 , indivíduos expostos à poeira ocasionada pelo desastre do World Trade Center, Estados Unidos 49,58, trabalhadores de agropecuária expostos a pó ${ }^{59}$, fazendeiros de plantações de milho e soja e criadores 
de porcos expostos à poeira decorrente de operações de confinamento de grãos e de animais 50, bem como em aborígenes expostos à poeira geogênica 51. Além disso, constatou-se associação entre PAIR e chumbo no sangue em trabalhadores da construção naval expostos à poeira de metais 52 . Salientase que, com exceção do estudo feito com aborígenes, em todos os demais estudos citados acima, os indivíduos estavam expostos também a outros riscos para alterações auditivas, tais como ruído e substâncias químicas.

O estudo de Santos et al. 9 constatou uma prevalência de handicap auditivo de 14,4\% em trabalhadores da construção civil, sendo que a exposição à poeira $(\mathrm{RP}=1,59)$ foi uma das associações encontradas. No estudo de Cone et al. ${ }^{58}$, a prevalência de perda auditiva aumentou conforme o aumento dos níveis de poeira, de 1,3\%, entre aqueles que não estavam na nuvem de poeira, para 2,2\%, para aqueles que estavam na nuvem de poeira, mas eram capazes de ouvir, até 5,7\%, para aqueles que estavam na nuvem de poeira, mas eram incapazes de ouvir. Os autores concluíram que efeitos adversos à saúde constatados nos sobreviventes do desastre do World Trade Center provavelmente foram resultantes dos efeitos combinados dos elevados níveis de ruído, da alta alcalinidade dos componentes do concreto e do gesso e da toxicidade das complexas misturas dos químicos constituintes.

Além dos tipos de poeiras citados acima, outros tipos têm sido relatados como possíveis fatores de risco para o desenvolvimento de alterações auditivas em trabalhadores, tais como poeira de chumbo ${ }^{7}$. A poeira de chumbo também foi considerada como causadora de alterações no sistema auditivo central, sem alterações nos limiares auditivos, de crianças que acidentalmente entraram em contato com ela por meio das roupas dos pais que trabalhavam em uma fábrica de baterias ou com a tinta descascada de suas casas 60 .

Nessa população, não foi evidenciada associação estatisticamente significante entre dificuldade auditiva e substâncias químicas. Esse achado vai de encontro às evidências de pesquisas recentes 11,61,62. Contudo, embora a variável substância química não tenha apresentado associação, reforça-se que a associação verificada com a poeira industrial não pode eliminar uma possível relação, visto que podem ser encontradas substâncias químicas nas partículas da poeira ${ }^{7}$. Ainda, na presente pesquisa, não foi possível considerar, nas análises, exposições combinadas a fatores de risco. Uma revisão sistemática com metanálise 42 concluiu que há chances significativamente maiores de trabalhadores adquirirem uma perda auditiva quando são expostos a uma combinação de solventes e ruído, em vez de apenas solventes. Além disso, sabe-se que a perda auditiva ocasionada por ruído e/ou substâncias químicas, em geral, afeta, inicialmente, as frequências mais altas da audição, o que pode passar despercebido pelos indivíduos no início de uma perda, podendo haver prevalência subestimada.

Além de possíveis danos ao sistema auditivo, as substâncias químicas também podem provocar intoxicação. No ano de 2017, o Sistema Nacional de Informações Tóxico-Farmacológicas (SINITOX) registrou 76.115 casos de intoxicação humana por agentes tóxicos, sendo que 5,53\% dos casos foram em ambiente ocupacional 63.

A perda auditiva também é resultado de um acúmulo de processos de exposição que envolvem atitudes individuais e mudanças ambientais aliadas ao processo do envelhecimento, já que a idade é o maior fator de risco para a perda auditiva 35. As transformações sociais têm influenciado os comportamentos de lazer, especialmente quando se trata do uso crescente de fones de ouvido. Imam \& Hannan 64 citaram que há risco em desenvolver uma perda auditiva permanente em exposição a níveis de ruído acima de $89 \mathrm{~dB}$ por mais de cinco horas por semana. Dessa forma, o conhecimento da população quanto aos riscos de exposições a ambientes e situações ruidosas torna-se essencial, como medida de prevenção, além de ressaltar outros fatores de risco modificáveis, como tabagismo, sedentarismo e diabetes 65

Apesar dos esforços voltados à implantação de programas de prevenção da perda auditiva ocupacional, como os programas de conservação auditiva, observa-se que os níveis de ruído reduzem no ambiente de trabalho, mas as medidas de controle, por voltarem-se majoritariamente para o uso dos equipamentos de proteção individual (EPI), ainda necessitam ser fortalecidas, tendo em vista sua efetividade apenas em curto prazo 66. A Saúde do Trabalhador, que envolve ações de assistência, promoção, vigilância e prevenção dos agravos, sofreu um avanço considerável a partir da consolidação das políticas públicas de atenção integral em Saúde do Trabalhador 67. Apesar das práticas em Saúde do Trabalhador no Sistema Único de Saúde (SUS) serem implementadas de forma lenta e com limitações, é possível observar avanços na compreensão e no enfrentamento dos desafios 68 . 
Faz-se necessário ressaltar que alguns elementos devem ser considerados ao interpretar os resultados desta pesquisa. $\mathrm{O}$ uso de medidas autorreferidas pode ser considerado uma limitação, sobretudo a medida de percepção auditiva. Contudo, apesar de o autorrelato apresentar limitações em função de seu caráter subjetivo, autores apontaram que a percepção auditiva possui elevados valores de sensibilidade e especificidade, podendo ser uma ferramenta confiável em estudos populacionais que, logisticamente, inviabilizam a realização de exames clínicos, como a audiometria tonal liminar 69. Além disso, no presente estudo, não foram considerados o uso de EPI e de aparelhos de amplificação sonora individual (AASI) nas análises, embora a prevalência de utilização de AASI entre os trabalhadores tenha sido pequena $(0,1 \%)(n=47)$. Com relação ao EPI, essa informação não estava disponível no banco de dados.

Como potencialidade, destaca-se a investigação das associações em uma amostra representativa do Brasil. Os métodos utilizados também são um ponto forte, ressaltando que os inquéritos de saúde se constituem em um importante método de coleta de dados epidemiológicos, capazes de embasar ações de promoção de saúde mais eficazes. Para o futuro, sugere-se a realização de pesquisas que incluam, em suas análises, exposições combinadas a agentes otoagressivos, além da recomendação da coleta de medidas objetivas, por meio de exames audiológicos, que possam avaliar tanto o sistema auditivo periférico quanto o central. Investigações acerca da relação entre perda de audição e agentes otoagressivos específicos também são indicadas.

Conclui-se que houve associação entre dificuldade auditiva autorreferida e exposição ocupacional a ruído e à poeira industrial em trabalhadores brasileiros. É necessário motivar gestores públicos a desenvolver e a implementar estratégias que tenham o ambiente ocupacional no centro das discussões. Dessa maneira, será possível promover a melhoria das condições de saúde dos trabalhadores.

\section{Colaboradores}

D. Hillesheim foi responsável pela concepção do artigo, revisou a literatura, redigiu a primeira versão, analisou os dados e aprovou a versão final para publicação. F. Zucki e S. M. Roggia colaboraram na redação, revisão de literatura e revisão final do manuscrito e aprovou a versão final para publicação. K. M. Paiva participou da análise dos dados, redação, revisão de literatura e aprovou a versão final para publicação.

\section{Informações adicionais}

ORCID: Danúbia Hillesheim (0000-0003-06004072); Fernanda Zucki (0000-0002-5473-9762); Simone Mariotti Roggia (0000-0003-0530-7530); Karina Mary de Paiva (0000-0001-7086-534X).

\section{Referências}

1. Vos T, Abajobir A, Abate K, Abbafati C, Abbas K, Abd-Allah F, et al. Global, regional, and national incidence, prevalence, and years lived with disability for 328 diseases and injuries for 195 countries, 1990-2016: a systematic analysis for the Global Burden of Disease Study 2016. Lancet 2017; 390:1211-59.

2. World Health Organization. Deafness and hearing loss. https://www.who.int/healthtopics/hearing-loss $\#$ tab $=$ tab_1 (acessado em Jun/2020).

3. World Health Organization. Deafness and hearing loss: key facts. https://www.who.int/ en/news-room/fact-sheets/detail/deafnessand-hearing-loss (acessado em Jun/2020).

4. Hillesheim D, Paiva KM, Rech CR, Vargas JCB, Luiza Neto I, Günther H, et al. Mobilidade urbana ativa de adultos com perda auditiva e a percepção sobre o ambiente: um estudo multicêntrico. Cad Saúde Pública 2019; 35:e00209418.

5. Heitz E, Gianattasio K, Prather C, Talegawkar S, Power M. Self-reported hearing loss and nonfatal fall-related injury in a nationally representative sample. J Am Geriatr Soc 2019; 67:1410-6.

6. von Gablenz P, Hoffmann E, Holube I. Prevalence of hearing loss in Northern and Southern Germany. HNO 2017; 65 Suppl 2:130-5. 
7. Johnson A-C, Morata TC. Occupational exposure to chemicals and hearing impairment. Gothenburg: University of Gothenburg; 2010.

8. Kim K, Kwon O. Prevalence and risk factors of hearing loss using the Korean Working Conditions Survey. Korean J Audiol 2012; 16:54-64.

9. Santos A, Silva A, Luccia G, Botelho C, Riva D. Desvantagem auditiva psicossocial e fatores associados em trabalhadores do setor da construção em Mato Grosso, Brasil. Rev Bras Epidemiol 2017; 20:501-13.

10. Ministério da Saúde. Portaria no 777, de 28 de abril de 2004. Dispõe sobre os procedimentos técnicos para a notificação compulsória de agravos à saúde do trabalhador em rede de serviços sentinela específica, no Sistema Único de Saúde - SUS. Diário Oficial da União 2004; 29 abr.

11. Lie A, Skogstad M, Johannessen H, Tynes T, Mehlum I, Nordby K, et al. Occupational noise exposure and hearing: a systematic review. Int Arch Occup Environ Health 2015; 89:351-72.

12. Sliwinska-Kowalska M, Davis A. Noise-induced hearing loss. Noise Health 2012; 14:274.

13. Carvalho L, Costa-Amaral I, Mattos R, Larentis A. Exposição ocupacional a substâncias químicas, fatores socioeconômicos e Saúde do Trabalhador: uma visão integrada. Saúde Debate 2017; 41:313-26.

14. Basner M, Babisch W, Davis A, Brink M, Clark C, Janssen S, et al. Auditory and non-auditory effects of noise on health. Lancet 2014; 383:1325-32.

15. Pawlaczyk-Luszczynska M, Dudarewicz A, Zamojska-Daniszewska M, Zaborowski K, Rutkowska-Kaczmarek P. Noise exposure and hearing status among call center operators. Noise Health 2018; 20:178-89.

16. Staudt A, Whitworth K, Chien L, Whitehead L, Gimeno Ruiz de Porras D. Association of organic solvents and occupational noise on hearing loss and tinnitus among adults in the U.S., 1999-2004. Int Arch Occup Environ Health 2019; 92:403-13.

17. Muhr P, Johnson A, Selander J, Svensson E, Rosenhall U. Noise exposure and hearing impairment in Air Force pilots. Aerosp Med Hum Perform 2019; 90:757-63.

18. Couth S, Mazlan N, Moore D, Munro K, Dawes P. Hearing difficulties and tinnitus in construction, agricultural, music, and finance industries: contributions of demographic, health, and lifestyle factors. Trends Hear 2019; 23:2331216519885571.

19. Cavalcante F, Ferrite S, Meira TC. Exposição ao ruído na indústria de transformação no Brasil. Rev CEFAC 2013; 15:1364-70.

20. Assunção AA, Abreu MNS, Souza PSN. Prevalência de exposição a ruído ocupacional em trabalhadores brasileiros: resultados da Pesquisa Nacional de Saúde, 2013. Cad Saúde Pública 2019; 35:e00094218.
21. Oenning N, Goulart B, Ziegelmann P, Chastang J, Niedhammer I. Associations between occupational factors and self-rated health in the national Brazilian working population. BMC Public Health 2019; 19:1381.

22. Soares M, Oenning N, Ziegelmann P, Goulart B. Association between self-reported hearing impairment and diabetes: a Brazilian population-based study. Arch Public Health 2018; 76:62.

23. Kobau R, Sniezek J, Zack MM, Lucas RE, Burns A. Well-being assessment: an evaluation of well-being scales for public health and population estimates of well-being among US adults. Applied Psychol Health Well-Being 2010; 2:272-97.

24. Tucker S, Diekrager D, Turner N, Kelloway EK. Work-related injury underreporting among young workers: prevalence, gender differences, and explanations for underreporting. J Safety Res 2014; 50:67-73.

25. Free H, Groenewold M, Luckhaupt S. Lifetime prevalence of self-reported work-related health problems among U.S. Workers - United States, 2018. MMWR Morb Mortal Wkly Rep 2020; 69:361-5.

26. Malta D, Szwarcwald C, Silva Júnior J. Primeiros resultados da análise do laboratório da Pesquisa Nacional de Saúde. Rev Bras Epidemiol 2019; 2 Suppl 2:E190001.

27. Szwarcwald C, Malta D, Pereira C, Vieira M, Conde W, Souza Júnior P, et al. Pesquisa Nacional de Saúde no Brasil: concepção e metodologia de aplicação. Ciênc Saúde Colet 2014; 19:333-42.

28. Souza-Júnior P, Freitas M, Antonaci G, Szwarcwald C. Desenho da amostra da Pesquisa Nacional de Saúde 2013. Epidemiol Serv Saúde 2015; 24:207-16.

29. Francisco P, Donalisio M, Barros M, Cesar C, Carandina L, Goldbaum M. Medidas de associação em estudo transversal com delineamento complexo: razão de chances e razão de prevalência. Rev Bras Epidemiol 2008; 11:347-55.

30. Rothman KJ, Greenland S, Lash TL. Modern epidemiology. 3a Ed. Philadelphia: Lippincott Williams \& Wilkins; 2008.

31. Medeiros AM, Assunção AA, Santos JN. Perda auditiva em trabalhadores do transporte urbano na Região Metropolitana de Belo Horizonte, Minas Gerais, Brasil. Cad Saúde Pública 2015; 31:1953-63.

32. Masterson E, Themann C, Luckhaupt S, Li J, Calvert G. Hearing difficulty and tinnitus among U.S. workers and non-workers in 2007. Am J Ind Med 2016; 59:290-300.

33. Kerns E, Masterson E, Themann C, Calvert G. Cardiovascular conditions, hearing difficulty, and occupational noise exposure within US industries and occupations. Am J Ind Med 2018; 61:477-91. 
34. Engdahl B, Tambs K. Occupation and the risk of hearing impairment - results from the Nord-Trøndelag study on hearing loss. Scand J Work Environ Health 2010; 36:250-7.

35. Joo Y, Hong $\mathrm{O}$, Wallhagen $\mathrm{M}$. The risk factors for age-related hearing loss: an integrative review. Ann Gerontol Geriatr Res 2016; 3:1039.

36. Fischer N, Weber B, Riechelmann H. Presbyakusis - altersschwerhörigkeit. Laryngorhinootologie 2016; 95:497-510.

37. Stanaway J, Afshin A, Gakidou E, Lim S, Abate $\mathrm{D}$, Abate $\mathrm{K}$, et al. Global, regional, and national comparative risk assessment of 84 behavioural, environmental and occupational, and metabolic risks or clusters of risks for 195 countries and territories, 1990-2017: a systematic analysis for the Global Burden of Disease Study 2017. Lancet 2018; 392:1923-94.

38. Cruz MS, Oliveira LR, Carandina L, Lima MCP, César CLG, Barros MBA, et al. Prevalência de deficiência auditiva referida e causas atribuídas: um estudo de base populacional. Cad Saúde Pública 2009; 25:1123-31.

39. Paiva KM, Cesar CLG, Alves MCGP, Barros MBA, Carandina L, Goldbaum M. Envelhecimento e deficiência auditiva referida: um estudo de base populacional. Cad Saúde Pública 2011; 27:1292-300.

40. Nelson DI, Nelson RY, Concha-Barrientos $\mathrm{M}$, Fingerhut $\mathrm{M}$. The global burden of occupational noise-induced hearing loss. Am J Ind Med 2005; 48:446-58.

41. Park H, Yoo M, Woo S, Kim S, Cho Y. Prevalence of hearing loss and associated factors in subjects with normal otoscopy: a national cross-sectional study. Int J Audiol 2017; 56:951-7.

42. Nakhooda F, Sartorius B, Govender S. The effects of combined exposure of solvents and noise on auditory function - a systematic review and meta-analysis. S Afr J Commun Disord 2019; 66:e1-e11.

43. Boéchat EM. Tratado de audiologia. 2a Ed. Rio de Janeiro: Editora Guanabara Koogan; 2015.

44. Kurabi A, Keithley E, Housley G, Ryan A, Wong A. Cellular mechanisms of noise-induced hearing loss. Hear Res 2017; 349:12937.

45. Le T, Straatman L, Lea J, Westerberg B. Current insights in noise-induced hearing loss: a literature review of the underlying mechanism, pathophysiology, asymmetry, and management options. J Otolaryngol Head Neck Surg 2017; 46:41.

46. Silva VAR, Mitre EI, Crespo AN. Is noise-induced hearing loss still a public health problem after decades of legislation? Braz J Otorhinolaryngol 2020; 86:6656.

47. Chen Y, Zhang M, Qiu W, Sun X, Wang X, Dong $Y$, et al. Prevalence and determinants of noise-induced hearing loss among workers in the automotive industry in China: a pilot study. J Occup Health 2019; 61:387-97.
48. Themann C, Masterson E. Occupational noise exposure: a review of its effects, epidemiology, and impact with recommendations for reducing its burden. J Acoust Soc Am 2019; 146:3879-905.

49. Stein C, Lee D, Flamme G, Cone J. Persistent post-9/11 hearing problems among World Trade Center health registry rescue and recovery workers, 2001 to 2007. J Occup Environ Med 2017; 59:1229-34.

50. Cramer M, Wendl M, Sayles H, Duysen E, Achutan C. Knowledge, attitudes, and practices for respiratory and hearing health among Midwestern farmers. Public Health Nurs 2016; 34:348-58.

51. Melody S, Bennett E, Clifford H, Johnston F, Shepherd C, Alach Z et al. A cross-sectional survey of environmental health in remote $\mathrm{Ab}$ original communities in Western Australia. Int J Environ Health Res 2016; 26:525-35.

52. Murata M, Costa-Amaral I, Carvalho L, Souza G, Mainenti H, Carvalho M, et al. Alterações respiratórias, auditivas e citogenéticas em trabalhadores de um estaleiro no Rio de Janeiro: estudo de caso. Cad Saúde Colet (Rio J.) 2017; 25:394-404.

53. Singh SB, Gautam S, Bhatta N, Shrestha G, Gautam R, Poudel S. Respiratory disorders among dust exposed workers. JNMA J Nepal Med Assoc 2019; 57:14-9.

54. Neitzel R, Andersson M, Lohman S, Sällsten G, Torén K, Andersson E. A semi-quantitative job exposure matrix for dust exposures in Swedish soft tissue paper mills. Am J Ind Med 2020; 63:359-67.

55. Barbosa H, Aguiar R, Bernardes H, Azevedo Junior R, Braga D, Szpilman A. Perfil clínico epidemiológico de pacientes com perda auditiva. J Health Biol Sci 2018; 6:424-30.

56. Nunes A, Silva C, Balen S, Souza D, Barbosa I. Prevalence of hearing impairment and associated factors in school-aged children and adolescents: a systematic review. Braz J Otorhinolaryngol 2019; 85:244-53.

57. Sayler S, Long R, Nambunmee K, Neitzel R. Respirable silica and noise exposures among stone processing workers in northern Thailand. J Occup Environ Hyg 2018; 15:117-24.

58. Cone J, Stein C, Lee D, Flamme G, Brite J. Persistent hearing loss among World Trade Center health registry residents, passersby and area workers, 2006-2007. Int J Environ Res Public Health 2019; 16:3864.

59. Haeffner R, Sarquis L, Heck R, Jardim V. Prevalence of hearing problems and associated factors in an agricultural company in southern Brazil. Rev Bras Epidemiol 2015; 18:679-90.

60. Otto D, Robinson G, Baumann S, Schroeder S, Mushak P, Kleinbaum D, et al. 5-Year followup study of children with low-to-moderate lead absorption: electrophysiological evaluation. Environ Res 1985; 38:168-86. 
61. Fábelová L, Loffredo C, Klánová J, Hilscherová K, Horvat M, Tihányi J, et al. Environmental ototoxicants, a potential new class of chemical stressors. Environ Res 2019; 171:378-94.

62. Roggia S, de França A, Morata T, Krieg E, Earl B. Auditory system dysfunction in Brazilian gasoline station workers. Int J Audiol 2019; 58:484-96.

63. Sistema Nacional de Informações Tóxico-farmacológicas, Fundação Oswaldo Cruz. Dados de intoxicação. Casos registrados de intoxicação humana por agente tóxico e circunstância. https://sinitox.icict.fiocruz.br/sites/sinitox. icict.fiocruz.br/files//Brasil4_1.pdf (acessado em Jun/2020).

64. Imam L, Hannan S. Noise-induced hearing loss: a modern epidemic? Br J Hosp Med (Lond) 2017; 78:286-90.

65. Ding T, Yan A, Liu K. What is noise-induced hearing loss? Br J Hosp Med (Lond) 2019; 80:525-9.
66. Tikka C, Verbeek J, Kateman E, Morata T, Dreschler W, Ferrite S. Cochrane method for systematic review and meta-analysis of interventions to prevent occupational noiseinduced hearing loss - abridged. Codas 2020; 32:e20190127.

67. Costa D, Lacaz F, Jackson Filho J, Vilela R. Saúde do trabalhador no SUS: desafios para uma política pública. Rev Bras Saúde Ocup 2013; 38:11-21.

68. Gomez C, Vasconcellos L, Machado J. Saúde do trabalhador: aspectos históricos, avanços e desafios no Sistema Único de Saúde. Ciênc Saúde Colet 2018; 23:1963-70.

69. Marini A, Halpern R, Aerts D. Sensibilidade, especificidade e valor preditivo da queixa auditiva. Rev Saúde Pública 2005; 39:982-4. 


\section{Abstract}

This study's objective was to estimate the association between self-reported hearing impairment and occupational exposure to hazardous noise and ototoxic agents in Brazilian workers. This was a cross-sectional study with data from the Brazilian National Health Survey (PNS-2013). The dependent variable was self-reported hearing impairment, and three principal exposures were analyzed: noise, industrial dust, and chemical substances. Logistic regression was performed, estimating crude and adjusted odds ratios (OR) with 95\% confidence intervals (95\%CI). The principal exposure variables were adjusted for each other and by covariables sex, age, workplace, time on the job, and hypertension. 36, 442 workers participated in the study. Higher prevalence of hearing impairment was seen in workers exposed to industrial dust (9.9\%) ( $p<0.001)$. The older the worker and the longer the time on the job, the higher the prevalence of hearing impairment $(p<0.001)$. In the adjusted analysis, workers exposed to noise showed 1.65 higher odds of reporting difficulty hearing, when compared to unexposed individuals ( $p<$ 0.001). The same was true for workers exposed to industrial dust $(O R=1.36)(p=0.012)$. No association was observed between the outcome and exposure to chemical substances $(p=0.120)$. There was an association between hearing impairment and occupational exposure to noise and industrial dust in Brazilian workers. This emphasizes the importance of strengthening public policies for hearing health and the development of measures for prevention and auditory monitoring in the workplace.

Hearing Loss; Occupational Health; Health Surveys; Noise; Dust

\section{Resumen}

El objetivo de este estudio fue estimar la asociación entre dificultad auditiva autoinformada $y$ la exposición ocupacional a agentes otoagresores en trabajadores brasileños. Se trata de un estudio transversal, realizado con datos de la Encuesta Nacional de Salud (PNS-2013). La variable dependiente fue la dificultad auditiva autoinformada y se analizaron tres exposiciones principales: ruido, polvo industrial y substancias químicas. Se realizó un análisis de regresión logística, estimándose las odds ratio (OR) brutas y ajustadas, con intervalos de un 95\% de confianza (IC95\%). Las variables principales de exposición fueron ajustadas entre sí y por las covariables: sexo, edad, ambiente de trabajo, tiempo de trabajo e hipertensión arterial. Participaron en este estudio 36.442 trabajadores. La mayor prevalencia de dificultad auditiva se observó en individuos expuestos al polvo industrial (9,9\%) ( $p<0,001)$. Asimismo, cuanto mayor era la edad del trabajador y tiempo de trabajo, mayor fue la prevalencia del desenlace $(p<0,001)$. En el análisis ajustado, los trabajadores expuestos a ruido presentaron 1,65 veces más oportunidad de informar de dificultad auditiva, cuando se comparan con los individuos no expuestos $(p<$ 0,001). Lo mismo sucedió con trabajadores expuestos a polvo industrial $(O R=1,36)(p=0,012)$. No se observó una asociación entre el desenlace y la variable exposición a sustancias químicas ( $p=$ 0,120 ). Se observó una asociación entre dificultad auditiva y exposición ocupacional a ruido y polvo industrial en trabajadores brasileños. Se refuerza la importancia del perfeccionamiento de políticas públicas en salud auditiva y desarrollo de acciones dirigidas a la prevención y monitoreo auditivo en el entorno laboral.

Pérdida Auditiva; Salud Laboral; Encuestas Epidemiológicas; Ruido; Polvo
Recebido em 10/Jul/2020

Versão final reapresentada em 26/Nov/2020

Aprovado em 15/Jan/2021 